\title{
Online Blind Calibration of Non-uniform Photodetectors: Application to Endomicroscopy
}

\author{
Nicolas Savoire, Barbara André, and Tom Vercauteren \\ Mauna Kea Technologies, Paris
}

\begin{abstract}
We present an original method for the online blind calibration of non-uniform photodetectors. The disparity of the detectors may arise from both irregular spatial arrangement and distinct slowly time-varying photometric transfer functions. As natural images are mostly continuous, the signal collected by neighboring detectors is strongly correlated over time. The core idea of our method is to translate the calibration problem into relative pairwise calibrations between neighboring detectors followed by the regularized inversion of a system akin to gradient-based surface recovery. From our blind calibration procedure, we design an online blind calibration pipeline compatible with clinical practice. Online blind calibration is proved to be statistically better than standard offline calibration for reconstructing endomicroscopy sequences.
\end{abstract}

\section{The Need for Online Blind Calibration in Endomicroscopy}

In vivo endomicroscopy consists of imaging the tissue at microscopic level, by inserting, through a standard endoscope, a probe made of tens of thousands of optical fibers. A laser scanning unit sends along each fiber an excitation light that is locally absorbed by fluorophores in the tissue and emitted back at a longer wavelength along the same fiber to a photodetector. Raw images are produced at a rate of 9 to 18 frames per second. The standard endomicroscopy image reconstruction scheme proposed by Le Goualher et al. [1] is composed of three steps: estimation of the signal collected by each fiber, compensation of calibration coefficients, and interpolation. Alternative less physically motivated methods [2, 3 use image filtering to remove the fiber honeycomb pattern at the cost of introducing some blur. As described in [1, each fiber $i$ of the endomicroscopy probe acts as a mono-pixel photodetector with an affine transfer function:

$$
v_{i}(t)=\alpha_{i} u_{i}(t)+\beta_{i}
$$

where $\alpha_{i}$ and $\beta_{i}$ are respectively the gain and offset of fiber $i, u_{i}(t)$ is the concentration of fluorophore seen at time $t$ by fiber $i$, and $v_{i}(t)$ is the signal collected at time $t$ by fiber detector $i$.

For the estimation of the calibration coefficients, Le Goualher et al. 1] proposed a offline non-blind calibration method, assuming constant gain and offset for each fiber. This method compensates for affine fiber transfer functions by 
previously acquiring two reference images, one in the air and one in a fluorophore solution, and then deducing from these two images a static offset and a static gain for each fiber. However, fiber coefficients are both slowly time-varying and medium-dependent. Indeed, due to photo-bleaching, autofluorescence signal slowly decreases with illumination time during the course of the procedure unless a long pre-illumination has been performed. In addition, fiber gains also depend on refractive indices and dispersive properties of the observed medium. For these reasons, image quality after the offline calibration may be affected by the presence of a veil on the image, i.e. a static noise pattern, which can be seen in all three images on the top of Fig. 3. Blindly recovering the parameters of nonuniform photodetectors is an ill-posed problem that has been scarcely addressed in the literature. In the field of astronomy, Kuhn et al. 4. proposed a blind calibration method that requires shifted images of the exact same scene, which can typically not be acquired on in vivo samples. The closest work is found in the field of computer vision. Weiss [5] presented an algorithm for blindly recovering intrinsic images from a sequence of natural scenes with different illumination, but only considered detectors on regular grids and with a single gain coefficient. To the best of our knowledge, this paper proposes the first online blind calibration of non-uniform mono-pixel photodetectors.

Our first objective is to design a blind method for the calibration of photodetectors having both irregular spatial arrangement and distinct photometric transfer functions. We then show how our blind calibration can be applied in an online manner to handle slowly time-varying transfer functions.

\section{Solving an Inverse Problem on Temporal Windows}

Let us first focus on static blind calibration. The core observation behind our method is that, when looking at physical objects, neighbor fibers nearly see the same object signal over time, so the collected signal from two neighbor fibers is strongly temporally correlated. This property arises from the mostly continuous nature of natural images, where edges form a set of zero measure and where the noise can be measured for example using total variation as introduced by Rudin et al. 6]. To translate this into mathematical terms, we capture the spatial relationship of the irregularly arranged photodetectors with a Delaunay triangulation applied to fiber locations, and express (11) in terms of a global function $U_{t}(\boldsymbol{p})$ which represents the concentration of fluorophore in the observed medium at position $\boldsymbol{p}$ and time $t$. Let $G=(V, E)$ the undirected graph associated with the triangulation. In our endomicroscopy probes the fiber pattern is pseudo hexagonal leading to $|E| \simeq 3|V|$. In (1), we substitute for $u_{i}(t)$ the expression $U_{t}\left(\boldsymbol{\phi}(t)+\boldsymbol{p}_{\boldsymbol{i}}\right)$, where $\boldsymbol{p}_{\boldsymbol{i}}$ is the spatial position of fiber $i$ relative to the center of probe distal end, and $\phi(t)$ is the spatial position of the probe center at time $t$. We then have:

$$
v_{j}(t)=\frac{\alpha_{j}}{\alpha_{i}}\left(v_{i}(t)-\beta_{i}\right)+\beta_{j}+\epsilon_{i j}(t)
$$

where $\epsilon_{i j}(t)=\left(\boldsymbol{p}_{\boldsymbol{j}}-\boldsymbol{p}_{\boldsymbol{i}}\right) \cdot \nabla U_{t}\left(\boldsymbol{p}_{\boldsymbol{i}}+\boldsymbol{\phi}(t)\right)+o\left(\left\|\boldsymbol{p}_{\boldsymbol{j}}-\boldsymbol{p}_{\boldsymbol{i}}\right\|\right)$ is a noise term whose distribution is sparse, with the natural assumption, similar to [6], that spatial 
variations of the concentration $U_{t}($.$) are almost always smooth. The signals col-$ lected by two neighboring fibers can thus be related by the affine relationship $v_{j}(t) \simeq a_{i j} v_{i}(t)+b_{i j}$, from which we derive two systems:

$$
\begin{gathered}
\left\{a_{i j}=\frac{\alpha_{j}}{\alpha_{i}}\right\}_{(i, j) \in E} \\
\left\{b_{i j}=\beta_{j}-\frac{\alpha_{j}}{\alpha_{i}} \beta_{i}\right\}_{(i, j) \in E}
\end{gathered}
$$

Interestingly, the affine coefficients $a_{i j}$ and $b_{i j}$ may conceptually be seen as the gradients of the fiber gain map and of the fiber offset map, respectively, except that the fibers are distributed on a non regular grid. Thus, the problem of recovering the calibration coefficients from the relative pairwise calibrations is akin to recovering a surface from a gradient field [7,8].

Our blind calibration method is designed in two steps. The first step performs linear regressions on a buffer of collected images to estimate the relative calibration coefficients $\left(a_{i j}, b_{i j}\right)$ for all $(i, j) \in E$. Once all regressions have been computed, the second step consists of solving the gain system (3) and the offset system (4), in order to recover the gain $\alpha_{i}$ and the offset $\beta_{i}$ of each fiber $i$.

\subsection{Pairwise Relative Calibrations of Photodetectors}

The first step of our blind calibration method consists of estimating relative calibration coefficients $a_{i j}$ and $b_{i j}$ between two observed signals $v_{i}(t)$ and $v_{j}(t)$ that are collected by neighboring fibers $(i, j)$ and sampled at times $t \in\{1, \ldots, m\}$ without loss of generality. From equation (2), we have: $v_{j}(t)=a_{i j} v_{i}(t)+b_{i j}+\epsilon_{i j}(t)$. Noticing that there is measurement error in both variables, an orthogonal linear regression [9] is more appropriate than ordinary least squares regression to estimate $a_{i j}$ and $b_{i j}$. In order to account for outliers resulting from the non-normality of $\epsilon_{i j}(t)$, the most common example being when an edge occurs between two neighbor fibers, we perform a robust orthogonal regression. For this purpose, we use M-estimators because they are deterministic and computationally competitive compared to other methods. We choose to perform an iteratively reweighted least squares fitting algorithm, which is at iteration $p$ :

$$
\hat{\boldsymbol{\theta}}_{i j}^{(p)}=\underset{\left\{\boldsymbol{\theta}_{i j} \mid \theta_{1_{i j}}^{2}+\theta_{2_{i j}}^{2}=1\right\}}{\arg \min } \sum_{t=1}^{m} z\left(r_{i j, t}^{(p-1)}\right)\left(\theta_{1_{i j}} v_{i}(t)+\theta_{2_{i j}} v_{j}(t)+\theta_{3_{i j}}\right)^{2}
$$

where $\boldsymbol{\theta}$ represents the regression coefficients, $z(x)=\rho^{\prime}(x) / x$ is the weight function and $r_{i j, t}^{(p)}=\theta_{1_{i j}}^{(p)} v_{i}(t)+\theta_{2_{i j}}^{(p)} v_{j}(t)+\theta_{3_{i j}}^{(p)}$ is the orthogonal residual at iteration $p$ associated with a sample at time $t$. By choosing the Tukey's biweight function for $\rho($.$) , we ensure that the residuals larger than a cutoff value c$ are eliminated. We set $c=4.6851 \hat{\sigma}$, where $\hat{\sigma}$ is the median absolute deviation of residuals. Our robust algorithm initialization sets initial slope to $s_{j} / s_{i}$ and initial intercept to $\mu_{j}-s_{j} \mu_{i} / s_{i}$, where $\mu_{i}$ and $s_{i}$ respectively denote the median and interquartile range of $\left\{v_{i}(t)\right\}_{1 \leq t \leq m}$. 
As the probe may contain dead fibers that do not transmit any light, any regression between these fibers and their neighbors is sterile. To overcome this issue, we decide to detect and reject regressions which are bad fits, based on a robust goodness-of-fit measure $g_{i j}$ for $(i, j) \in E$. We define $g_{i j}$ as the weighted squared Pearson correlation coefficient between $v_{i}$ and $v_{j}: g_{i j}=$ $\left(\sum_{t=1}^{m} z_{i j, t}\left(v_{i}(t)-\overline{v_{i}}\right)\left(v_{j}(t)-\overline{v_{j}}\right)\right)^{2} /\left(\sum_{t=1}^{m} z_{i j, t}\left(v_{i}(t)-\overline{v_{i}}\right)^{2} \sum_{t=1}^{m} z_{i j, t}\left(v_{j}(t)-\right.\right.$ $\left.\left.\overline{v_{j}}\right)^{2}\right)$, where $\left\{z_{i j, t}\right\}_{1 \leq t \leq m}$ are the final weights of (5) after convergence, and $\overline{v_{i}}=\left(\sum_{t=1}^{m} z_{i j, t} v_{i}(t)\right) /\left(\sum_{t=1}^{m} z_{i j, t}\right)$. These $\left\{g_{i j}\right\}_{(i, j) \in E}$ will be used in the second step of our blind calibration method, focusing on system inversion.

\subsection{From Relative Calibrations to Global Calibration}

Gain Estimation. The gain system (3) uses the results of an linear orthogonal regression. The symmetry of orthogonal regression implies that noise on the estimates $\hat{a}_{i j}$ is better modeled as multiplicative. Therefore, (3) can be advantageously transposed to the log domain: $\left\{\alpha_{j}^{*}-\alpha_{i}^{*}=\hat{a}_{i j}^{*}\right\}_{(i, j) \in E}$ with $\alpha_{i}^{*}=\log \alpha_{i}$ and $\hat{a}_{i j}^{*}=\log \hat{a}_{i j}$. This can be rewritten as $M \boldsymbol{\alpha}^{*}=\hat{\boldsymbol{a}}^{*}$, where $M$ is the $|E| \times|V|$ matrix such as $M_{l p}=\delta_{p, j_{l}}-\delta_{p, i_{l}}$, with $E=\left\{\left(i_{l}, j_{l}\right), 1 \leq l \leq|E|\right\}$. In order to cope with noise and dead fibers, we introduce the weight function $w(x)=\left(1+e^{-\left(x-g_{0}\right)}\right)^{-1} \delta\left(x \geq g_{0}\right)$, where $\delta$ is the Kronecker operator. This function puts more weight on the relations of (3) associated with higher goodnessof-fit values, and ignores those associated with values below an arbitrary cutoff value $g_{0}=0.6$. To cope with the non-uniqueness of the solution and regularize the system, we add the a priori that the gains are close to 1 . We use the $|E| \times|E|$ diagonal matrix $W$ defined by $W_{l l}=w\left(g_{i_{l} j_{l}}\right)$ to obtain the weighted system:

$$
\hat{\boldsymbol{\alpha}}^{*}=\underset{\boldsymbol{\alpha}^{*}}{\arg \min }\left\|W\left(M \boldsymbol{\alpha}^{*}-\hat{\boldsymbol{a}}^{*}\right)\right\|^{2}+\lambda\left\|\boldsymbol{\alpha}^{*}\right\|^{2}
$$

The overdetermined system (6) is solved using a conjugate gradient method (LSQR) which has a good numerical stability for ill-conditioned systems. Finally, we deduce the estimated gains $\hat{\boldsymbol{\alpha}}=\exp \hat{\boldsymbol{\alpha}}^{*}$.

Offset Estimation. In order to uncouple offset estimation from gain estimation, we approximate $\alpha_{j} / \alpha_{i}$ by $\hat{a}_{i j}$ in the offset system (4). As (4) uses the estimates $\hat{b}_{i j}$ resulting from the symmetric orthogonal regression, we symmetrize the offset system by introducing a normalization factor $\gamma_{i j}=\left(1+\hat{a}_{i j}{ }^{2}\right)^{0.5}: \beta_{j} / \gamma_{i j}-\left(\hat{a}_{i j} / \gamma_{i j}\right) \beta_{i}=$ $\hat{b}_{i j} / \gamma_{i j}$. In order to regularize the system, we make the assumption $\mathcal{A}$ that fiber background $\boldsymbol{\beta}(t)$ at time $t>t_{0}$ can be approximated by $q \boldsymbol{\beta}_{0}$ where $\boldsymbol{\beta}_{0}$ is an initial offline background estimation and $q$ is an unknown global factor only depending on $t$ [10. Injecting $\beta_{i}=q \beta_{0_{i}}$ in (4) gives: $q \beta_{0_{j}}-\hat{a}_{i j} q \beta_{0_{i}} \simeq b_{i j}$. A robust estimate of $q$ is then: $\hat{q}=\operatorname{median}_{(i, j) \in E}\left\{\hat{b}_{i j} /\left(\beta_{0_{j}}-\hat{a}_{i j} \beta_{0_{i}}\right)\right\}$. From this estimate $\hat{q}$, we obtain an approximation $\hat{q} \boldsymbol{\beta}_{0}$ of $\boldsymbol{\beta}$ and use this value to regularize the system (4). Weighting the system as described in 2.2. offset estimation is rewritten as:

$$
\hat{\boldsymbol{\beta}}=\underset{\boldsymbol{\beta}}{\arg \min }\left\|W\left(A \boldsymbol{\beta}-\frac{1}{\boldsymbol{\gamma}} \hat{\boldsymbol{b}}\right)\right\|^{2}+\lambda\left\|\boldsymbol{\beta}-\hat{q} \boldsymbol{\beta}_{0}\right\|^{2}
$$


where $A$ is the $|E| \times|V|$ matrix such as $A_{l p}=\left(\delta_{p, j_{l}}-\delta_{p, i_{l}} \hat{a}_{i_{l} j_{l}}\right) / \gamma_{i_{l} j_{l}}$. Being similar to system (6), the system (7) is solved using LSQR.

\subsection{Online Blind Calibration Pipeline}

Our second objective is to integrate the blind calibration method described in Section 2.2 into an online calibration pipeline, illustrated in Fig. 2. For this purpose, we leverage the fact that the temporal variation of fiber transfer functions are quite slow compared to the temporal variation of fluorescence signal which is due to either motion within the tissue, or motion of the probe along the tissue during endomicroscopy procedure. We thus decide to apply our blind calibration method on temporal windows where transfer functions do not significantly change, and to perform successive calibration updates as soon as possible. At startup of the imaging system, the probe is in the air and laser illumination allows to record the non-fluorescent background signal $\boldsymbol{\beta}_{0}$. Standard offline calibration can be used as initial calibration. When the physician starts the acquisition at time $T_{0}$, successive frames are accumulated in a buffer until there is sufficient data to make system inversion possible. Our intuition is that the more moving fluorescent signal there is, the better linear regression results are, and so the more chance there is to solve the system. We consider that there is sufficient data if the following criterion $\mathcal{C}$ is satisfied: $\left|\left\{(i, j) \in E, g_{i j} \geq g_{0}\right\}\right| \geq 0.9|E|$. Regressions are performed until time $T_{1}$ when $\mathcal{C}$ is satisfied, then we perform system inversion and proceed to the first calibration update. At $k^{\text {th }}$ calibration update, buffer is flushed at $t=T_{k}+1$ and new frames are accumulated in the buffer from $t=T_{k}+1$ to $t=T_{k+1}$ when $(k+1)^{\text {th }}$ calibration is possible. From the gains $\hat{\boldsymbol{\alpha}}^{T_{k}}$ and the offsets $\hat{\boldsymbol{\beta}}^{T_{k}}$ estimated at time $T_{k}$, we construct the new calibrated signal as follows: $\left.\forall t \in] T_{k}, T_{k+1}\right], \hat{u}_{i}(t)=\left(v_{i}(t)-\hat{\beta}_{i}{ }^{T_{k}}\right) / \hat{\alpha}_{i}{ }^{T_{k}}$. This provides an estimate of the fluorophore concentration $u_{i}(t)$ seen by fiber $i$ at time t. Finally, interpolation of $\hat{\boldsymbol{u}}$ is performed.

\section{$3 \quad$ Evaluation and Results}

Before method evaluation, we aim at validating the assumption $\mathcal{A}: \boldsymbol{\beta}=q \boldsymbol{\beta}_{0}$. For this purpose, we tested 4 probes having different numbers of fibers and different optical properties. Each probe was used to acquire every minute a 10frame average image in the air. Laser illumination was continuously on during the first 14 minutes in order to measure autofluorescence decrease. After 14 minutes, to highlight the phenomenon of autofluorescence recovery, illumination was off except during acquisition of the images. We then calculated the Pearson correlation between the background signal of all fibers at time $t$ and those at time $t_{0}=0$. The results presented in Fig. 1 on the right, reveal that, for all 4 probe models the Pearson correlation coefficient is quite high, being superior to 0.98 , therefore validating $\mathcal{A}$.

Our database used for method evaluation is composed of 89 endomicroscopy sequences acquired during clinical procedures in 6 different medical centers. Each 

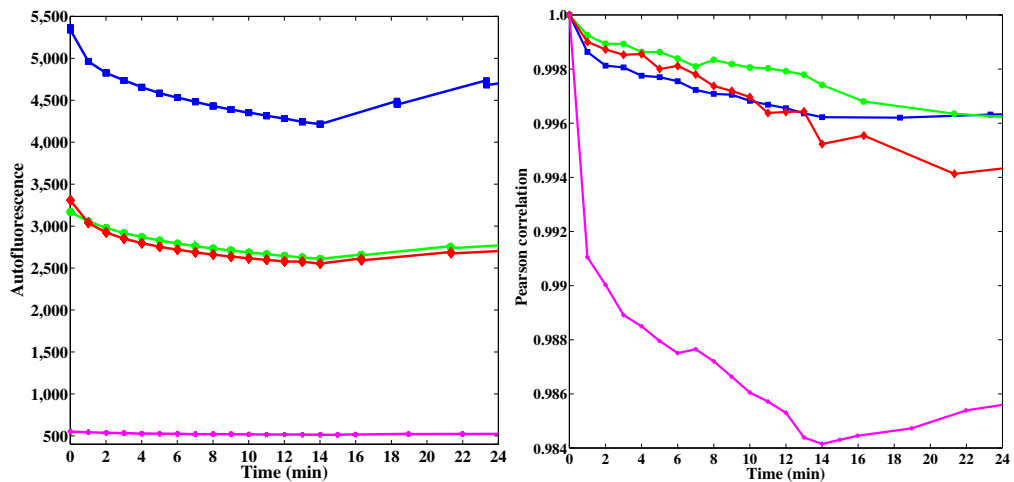

Fig. 1. Left: Autofluorescence decrease $(t \leq 14 \mathrm{~min})$ and recovery $(t>14 \mathrm{~min})$, measured for 4 different probe models. Right: Corresponding Pearson correlation values between fiber signal at time $t$ and at time $t_{0}$.

of these 89 sequences is complete in the sense that every single image from startup to shutdown was recorded. We propose to evaluate first the ability of our calibration method to recover the model parameters, then the impact of the calibration on image reconstruction. Since it is impossible to get an uniform medium with the same physical properties as a given observed living tissue, validation cannot rely on flat-field images.

Our online pipeline implies that calibration applied on current data is only computed from past data. This ensures unbiased performance evaluation on reconstructed data. Given a temporal window $\left.] T_{k}, T_{k+1}\right]$ where the calibration is static, we define a static calibration quality $C Q_{k}$ which measures the ability to align the calibrated gains of two neighboring fibers from the model parameters estimated on $\left.] T_{k-1}, T_{k}\right]: C Q_{k}=\left|\left\{(i, j) \in E, 1-\eta<\hat{a}_{i j}^{T_{k+1}} \cdot \hat{\alpha}_{i}^{T_{k}} / \hat{\alpha}_{j}^{T_{k}}<(1+\eta)^{-1}\right\}\right| /|E|$, where $\hat{a}_{i j}^{T_{k+1}}$ is the relative calibration estimated between fiber $i$ and fiber $j$ on the temporal window $\left.] T_{k}, T_{k+1}\right]$, and $\eta$ is an arbitrary threshold set to 0.1 . The expression $\hat{a}_{i j}^{T_{k+1}} \cdot \hat{\alpha}_{i}^{T_{k}} / \hat{\alpha}_{j}^{T_{k}}$ is basically an unbiased estimation of the residuals of (6). $C Q_{k}$ therefore provides an unbiased measure of how good we would be at reconstructing a flat image from a flat signal with the same physical properties as the observed medium. We then define the global calibration quality $C Q$ of the whole video sequence as the average of static calibration quality values.

According to the results presented in Fig. 2 on the left, online blind calibration yields statistically higher calibration quality than offline calibration $(95 \%$ confidence interval of $[0.13,0.19]$ using paired difference $t$-test). Visual inspection of worst cases revealed that the sequences for which offline calibration performs similarly or slightly better contains either mostly noise, static fluorescence signal, non-sufficient moving fluorescent signal or only thin fluorescent structures. For all 89 sequences, the first online calibration succeeded after less than 30 seconds of moving fluorescent signal, a delay which is clearly compatible with 
clinical practice. Regarding the sequences acquired with a probe of $n=10,000$ fibers for example, computation time was less than $4 \mathrm{~ms}$ per frame in the buffer for performing all regressions, and less than $300 \mathrm{~ms}$ for system inversion.

We then propose to evaluate the impact of the calibration on image reconstruction by measuring the noise (static and dynamic) in a calibrated sequence. For this purpose, we compute the average of the (Delaunay-based) total variation [6] on all $T$ frames of the sequence: $\sum_{t=1}^{T} \sum_{(i, j) \in E}\left|\hat{u}_{i}(t)-\hat{u}_{j}(t)\right| /(|E| T)$. Results show that the measured noise is statistically lower after online blind calibration than after offline calibration (95\% confidence interval of $[-55,-33]$ using paired difference $t$-test). The improvement of image quality, from offline
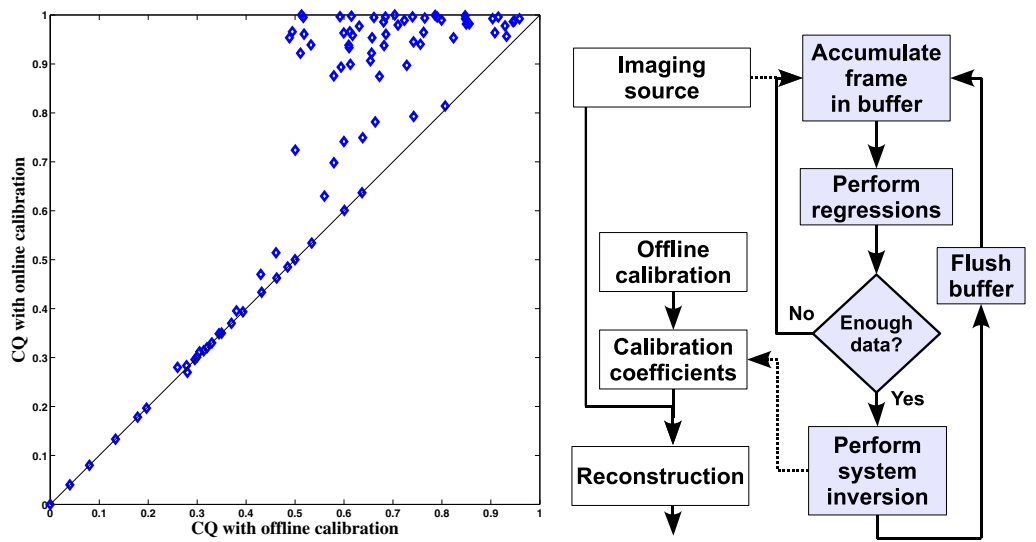

Fig. 2. Left: Calibration Quality (CQ) of 89 sequences processed by online versus offline calibration. Right: Pipeline of online blind calibration, highlighted in gray boxes. Dotted arrows show source input (top) and calibration update (bottom).
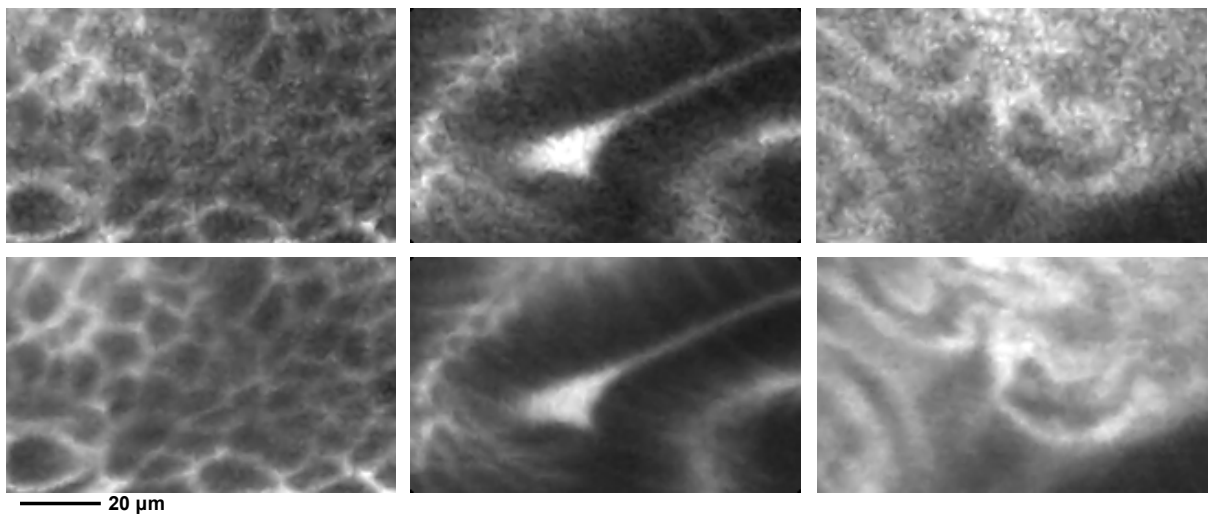

Fig. 3. Endomicroscopy image portions of Barrett's Esophagus, from 3 sequences processed by offline calibration (top) or online calibration (bottom). Bottom images appear much less noisy although no spatial smoothing is performed by online calibration. 
to online calibration, can be qualitatively appreciated on still images presented in Fig. 3, and on two video sequences acquired on the bile duct and on the colon, available as Supplemental Material (http://youtu.be/1WYEQysDBqQ).

\section{Conclusion}

We have presented an original method for online blind calibration of non-uniform photodetectors, where only past signal is used to calibrate current signal in a transparent way to the user. By performing robust linear regressions and regularizing an ill-posed inverse problem, our method is able to handle photodetectors having both irregular spatial arrangement and individual slowly time-varying photometric transfer functions. Using a relatively large database of complete sequences acquired during clinical endomicroscopy procedures, we have demonstrated that online blind calibration statistically outperforms standard offline calibration. For future work, we plan to evaluate whether online blind calibration leads to higher perceived image quality and better diagnostic performance for the physicians.

\section{References}

1. Le Goualher, G., Perchant, A., Genet, M., Cavé, C., Viellerobe, B., Berier, F., Abrat, B., Ayache, N.: Towards Optical Biopsies with an Integrated Fibered Confocal Fluorescence Microscope. In: Barillot, C., Haynor, D.R., Hellier, P. (eds.) MICCAI 2004. LNCS, vol. 3217, pp. 761-768. Springer, Heidelberg (2004)

2. Winter, C., Rupp, S., Elter, M., Münzenmayer, C., Gerhäuser, H., Wittenberg, T.: Automatic adaptive enhancement for images obtained with fiberscopic endoscopes. IEEE Trans. Biomed. Eng. 53(10), 2035-2046 (2006)

3. Elter, M., Rupp, S., Winter, C.: Physically motivated reconstruction of fiberscopic images. In: Proc. ICPR 2006, vol. 3, pp. 599-602 (August 2006)

4. Kuhn, J.R., Lin, H., Loranz, D.: Gain calibrating nonuniform image-array data using only the image data. In: Proc. ASPC 1991, vol. 103, pp. 1097-1108 (1991)

5. Weiss, Y.: Deriving intrinsic images from image sequences. In: Proc. ICCV 2001, vol. 2, pp. 68-75 (2001)

6. Rudin, L.I., Osher, S., Fatemi, E.: Nonlinear total variation based noise removal algorithms. Phys. D 60(1-4), 259-268 (1992)

7. Pérez, P., Gangnet, M., Blake, A.: Poisson image editing. ACM Trans. Graph. 22(3), 313-318 (2003)

8. Agrawal, A., Chellappa, R., Raskar, R.: An algebraic approach to surface reconstruction from gradient fields. In: Proc. ICCV 2005, vol. 1, pp. 174-181 (2005)

9. Markovsky, I., Van Huffel, S.: Overview of total least-squares methods. Signal Processing 87(10), 2283-2302 (2007)

10. Berglund, A.J.: Nonexponential statistics of fluorescence photobleaching. J. Chem. Phys. 121(7), 2899-2903 (2004) 